\title{
A FORMAL APPROACH TO DETECTING SHILLING BEHAVIORS IN CONCURRENT ONLINE AUCTIONS
}

\author{
Yi-Tsung Cheng and Haiping $\mathrm{Xu}$ \\ Computer and Information Science Department \\ University of Massachusetts Dartmouth \\ North Dartmouth, MA 02747 \\ Email: \{g_ycheng,hxu\}@umassd.edu
}

Keywords: Concurrent online auctions, Shilling behaviors, Model checking, Linear temporal logic (LTL).

\begin{abstract}
Shilling behaviors are one of the most serious fraud problems in online auctions, which make winning bidders have to pay more than what they should pay for auctioned items. In concurrent online auctions, where multiple auctions for the same type of items are running simultaneously, shilling behaviors can be even more severe because detecting, predicting and preventing such fraudulent behaviors become very difficult. In this paper, we propose a formal approach to detecting shilling behaviors in concurrent online auctions using model checking techniques. We first develop a model template that represents two concurrent online auctions in Promela. Based on the model template, we derive an auction model that simulates the bidding processes of two concurrent auctions. Then we use the SPIN model checker to formally verify if the auction model satisfies any questionable behavioral properties that are written in LTL (Linear Temporal Logic) formulas. Thus, our approach simplifies the problem of searching for shilling behaviors in concurrent online auctions into a model checking problem. Finally, we provide a case study to illustrate how our approach can effectively detect possible shill bidders.
\end{abstract}

\section{INTRODUCTION}

In traditional economic theory, auction can be used to determine the value of a commodity that is difficult to tag a price. The commodity can be a physical product, such as artwork and antiques; or it can be a virtual product, for example, spectrum licenses and procurement contracts. The most commonly used types of auctions include English auction, Dutch auction, sealed first-price auction, and sealed second-price auction. Among them, only English auction is adopted in online auction houses. In an English auction, participants can openly observe other people's bids and then bid against each other. The following bidding price must be higher than the previous one. The auction ends when the bidding reaches a point where no one wants to beat the current highest price. So, in an English auction, a bidder can bid multiple times while the bidding price ascends. The seller of the auctioned item can set a pre-determined reserve price. If the final bidding price is lower than the reserve price, the seller can reserve the right of not selling the auctioned item.
The characteristic of multiple bids and ascending bidding price in English auctions has made this auction type very popular, but it also makes shilling behaviors very common in online auctions.

There are two main kinds of shilling behaviors: reserve price shilling and competitive shilling (Kauffman and Wood, 2000). In reserve price shilling, a seller sets a low reserve price and pretends to be normal bidders to put in bids, in order to drive up the bidding price to his own evaluation of the item. Usually the lower reserve price the seller sets the cheaper fee he has to pay to the auction house. In this case, the seller can avoid paying higher reserve price fee. In competitive shilling, a seller also pretends to be normal bidders, and constantly monitors the bidding process and puts in fake bids to drive up the bidding price; however, the objective of doing this is to make potential buyers pay extra money to win their bids instead of paying less reserve price fee. Although the objectives of these two behaviors are different, their distinction is not always very clear. For example, a reserve-priceshilling seller might still want to drive up the bidding price, even after it has already reached the 
seller's own evaluation of the item. Notice that normally the reserve price shilling only affects the auction houses; while the competitive shilling affects all bidders in the market. It is obvious that the competitive shilling causes a greater harm to the auction market than the reserve price shilling. In addition, shilling behaviors involved in concurrent online auctions, where multiple auctions for the same type of items are running simultaneously, are much more difficult to detect than shilling behaviors occur in a standalone auction. Thus, in this paper, we focus on studying competitive shilling behaviors in concurrent online auctions.

There is very little previous work on shill detection for online auctions. Wang and his colleagues showed that private value English auctions with shill bidding can result in a higher expected seller profit than other auction formats (Wang et al., 2002). This explains why in online auction houses like eBay, shilling behaviors have become a very serious problem that cannot be ignored. The authors proposed a commission fee mechanism in which the auctioneer charges the seller a commission fee based on the difference between the winning bid and the seller's reserve price. This approach can make shill bidding unprofitable, but it could be unfair to sellers' interests, especially when the sellers are not shilling at all.

Kauffman and Wood used a statistical approach to detecting shilling behaviors and showed that how the statistic data of a market would look like if opportunistic behaviors do exist. They also showed how to use an empirical model to test for questionable behaviors (Kauffman and Wood, 2000). However, their approach suffers from a few problems, for example, it needs to review multiple auctions over a long period of time (Gupta and Bapna, 2002). Furthermore, the statistical approach could not deal with the shilling problem directly.

In this paper, we propose to use model checking technique to detect shilling behaviors in two concurrent online auctions. Our approach can directly detect shilling behaviors based on the latest auction data, and then suggest shill suspects, if any.

The rest of this paper is organized as follows: Section 2 introduces the pattern-based model checking technique. Section 3 first presents a motivation example for shill detection using model checking. Then it introduces a model template and shows how to build an auction model based on auction data from two concurrent auctions. Section 4 provides a case study for how to use our approach to detect shilling behaviors. Finally, in Section 5, we provide conclusions and our future work.

\section{PATTERN-BASED MODEL CHECKING TECHNIQUE}

\subsection{The SPIN Model Checker}

There is a wide variety of model checking tools available, such as SPIN, NuSMV2, Java Pathfinder and MARIA. Among them, the SPIN model checker provides a friendly user interface and accepts model specifications written in Promela (PROcess MEta Language) (Holzmann, 1997). Promela is a CSP-like language mainly used to describe abstract level concurrent software system. Like any other programming languages, Promela supports variables, arrays and user-defined data types as well as control flow statement. In addition, Promela supports symbolic constants, message channels, processes, selection and repetition, atomic sequence and deterministic steps.

\subsection{LTL and Patterns}

The SPIN model checker supports specification of system properties using Linear Temporal Logic (LTL) (Pnueli, 1977), which is a formal method to specify temporal relationships of statements. LTL has been proven to have good expressivity and more natural language like statements for verification. LTL consists only a few logic operators, such as $G$ (always), $F$ (eventually), $U$ (until), $W$ (unless, or weak until) and $O$ (next). Combining with Boolean operators, i.e., \&\& (and), \| (or), ! (negation), $\rightarrow$ (logical implication) and $\leftrightarrow$ (logical equivalence), LTL is capable of describing many key properties of a concurrent software system.

On the other hand, like many other formal specification and verification methods, writing a LTL formula is not easy and error prone. Even a person who has expertise in LTL may still have a difficult time in understanding the semantic of a LTL formula, such as []$((\mathrm{Q} \& \& ! \mathrm{R} \& \&<>\mathrm{R}) \rightarrow(\mathrm{P} \rightarrow$ $(! R U(S \& \& ! R))) U R)$. To solve this problem, Dwyer and his colleagues proposed a pattern-based approach to helping software engineers to specify requirements properties without having to worry about the complexity and potential traps (Dwyer et al., 1999).

There are quite a few patterns proposed in previous work (Dwyer et al., 1999). Before we present some of the patterns that we use in this paper, we first introduce a notation called pattern scope, which represents the extent of a program execution over which the pattern must hold. 


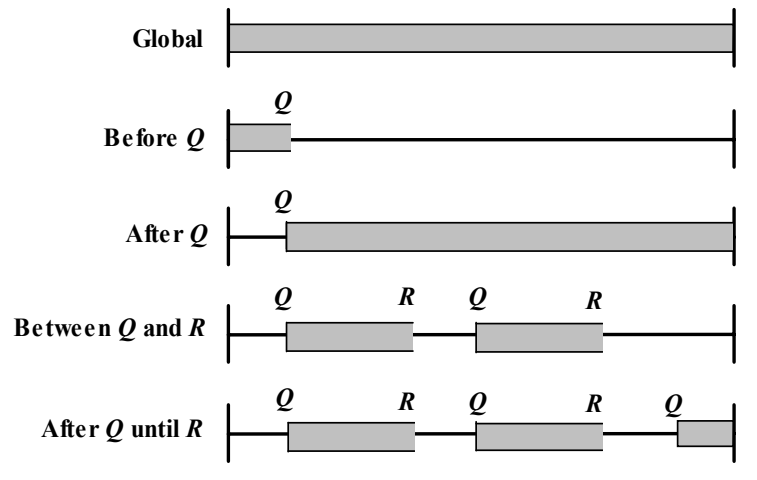

Figure 1: Pattern scopes for pattern-based LTL.

Figure 1 is an illustration of pattern scopes adapted from (Dwyer et al., 1999). The capital letters $Q$ and $R$ stand for events. Every pattern can be assigned with one of the five scopes, in which during the extent of the specified scope, a pattern must hold. It should be clarified that all these pattern scopes are defined as closed-left and open-right. For example, if the scope is "Between $Q$ and $R$ ", then $Q$ is included in the scope but $R$ is excluded.

In Table 1 and Table 2, we list two patterns that are used in this paper. For example, the Absence pattern in pattern scope "Before $R$ " is described by the formula $<>R \rightarrow(! P \quad U \quad R)$. It specifies that during the extent of the starting state and event $R$, event $P$ must be false. Similarly, the Existence pattern in pattern scope "Between $Q$ and $R$ " is described by the formula []$(\mathrm{Q} \& \& \quad \mathrm{R} \rightarrow(! \mathrm{R} W \quad(\mathrm{P}$ $\& \&(\mathrm{R}))$ ), which specifies that during the extent of event $Q$ and event $R$, event $P$ must become true.

For more LTL pattern definitions, please refer to previous work (Dwyer et al., 1999).

Table 1: Absence patterns (event $\mathrm{P}$ is false).

\begin{tabular}{|c|c|}
\hline Pattern Scope & Formula \\
\hline \begin{tabular}{|l|} 
Globally \\
\end{tabular} & {[]$(! P)$} \\
\hline Before $R$ & $<>R \rightarrow(! P \quad U \quad R)$ \\
\hline After $Q$ & {[]$(\mathrm{Q} \rightarrow[](! \mathrm{P}))$} \\
\hline Between $Q$ and $R$ & 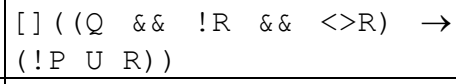 \\
\hline After $Q$ until $R$ & 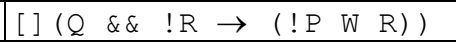 \\
\hline
\end{tabular}

Table 2: Existence patterns (event $\mathrm{P}$ becomes true).

\begin{tabular}{|c|c|}
\hline Pattern Scope & Formula \\
\hline Globally & $<>(\mathrm{P})$ \\
\hline Before $R$ & $! R \quad W \quad(P \quad \& \& \quad ! R)$ \\
\hline After $Q$ & []$(! Q Q) \quad|| \quad<>(Q \quad \& \& \quad<>P))$ \\
\hline Between $Q$ and $R$ & 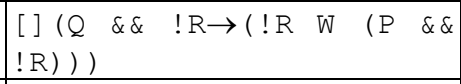 \\
\hline After $Q$ until $R$ & $\begin{array}{lll}] & \left(\begin{array}{ll}Q & \& \&\end{array} \quad ! \mathrm{R} \rightarrow\left(\begin{array}{lll}\mathrm{R} & \mathrm{U} & (\mathrm{P} \\
\& \& & (\mathrm{R}))\end{array}\right)\right. \\
\end{array}$ \\
\hline
\end{tabular}

\section{MODELING CONCURRENT ONLINE AUCTIONS}

\subsection{A Motivation Example}

The basic idea of our approach is to automatically generate an auction model based on auction data from two concurrent auctions, and verify if the auction model satisfies certain bidders' behavioral properties. The following figure (Figure 2) shows an example of two concurrent auctions.

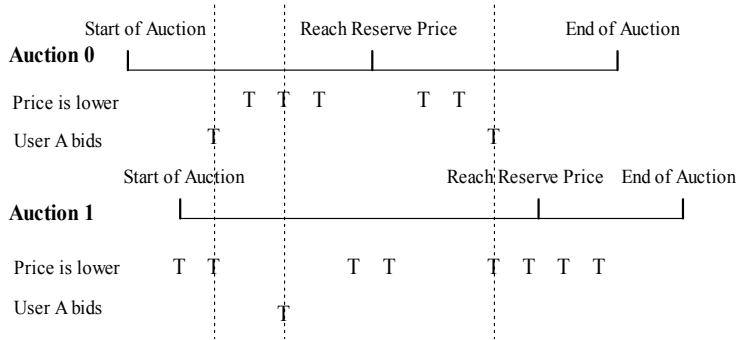

Figure 2: An example of two concurrent auctions.

To simplify matters, we assume that the auction that starts first is always Auction 0, and the one that starts later is always Auction 1. In Figure 2, "T" stands for "True", and for each auction it has two predicates, i.e., "Price is lower" and "User A bids". If any predicate becomes " $T$ " at a certain point of time in the process of any one of the two auctions, it means that the event happens at that time. For example, if "Price is lower" is " $T$ " at a point in Auction 0, it means that at that time, the bidding price is lower in Auction 0. Similarly, if "User A bids" is " $\mathrm{T}$ " at a point in Auction 1, it means that at that time User A puts in his/her bid in Auction 1.

We use an example to show how to write a pattern-based LTL formula for a certain behavioral property. For instance, we want to detect the following shilling behavior:

While two auctions are running concurrently, a user bids in the auction that has higher bidding price rather than lower bidding price.

Suppose Auction 0 starts first and also ends first. Then we need to verify the following: after "start of Auction 1" until "end of Auction 0", does "(User A bids in Auction 0 \&\& price is lower in Auction 1) or (User $A$ bids in Auction 1 \&\& price is lower in Auction 0) become true?" The formula can be composed using the Existence pattern with "After $Q$ until $R$ " scope. If we use "S1" to represent "start of Auction 1", "E0" to represent "end of Auction 0", "P" to represent "User $A$ bids in Auction 0 \&\& price is lower in Auction 1", and " $\mathrm{S}$ " to represent "User A bids in Auction 1 \&\& price is lower in Auction 0", 
the formula can be written as ( [ ] (SI \&\& !EO $\rightarrow$ $(! \mathrm{EO} U(\mathrm{P} \& \& \quad \mathrm{EO}))))$ | | ([] (SI \&\& !EO $\rightarrow$ $($ !EO U (S \&\& !EO)))) ).

From Figure 2, we can see that the behavior specified above becomes true for three times (denoted by three vertical dotted lines). Thus, the LTL formula must be valid.

\subsection{Preprocessing the Auction Data}

The first step to build an auction model is to preprocess the auction data. As shown in Figure 3, this task is accomplished by the Preprocessor, which extracts numeric data from two concurrent auctions and substitutes them into a model template to produce a specific auction model.

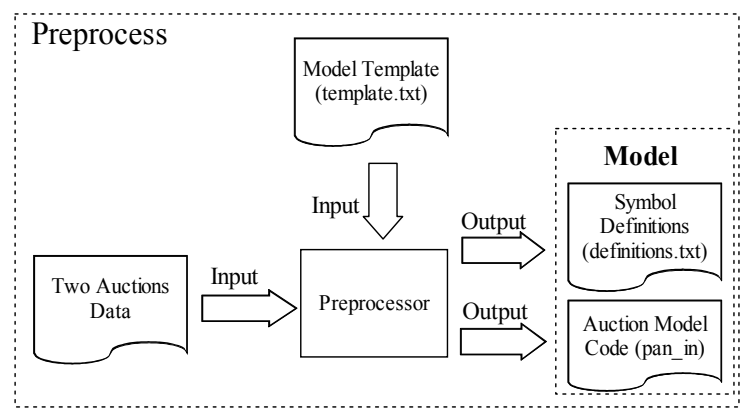

Figure 3: Preprocessing the auction data.

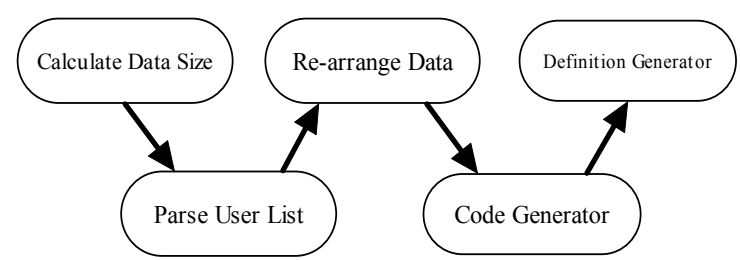

Figure 4: Preprocessor's major tasks.

The generated auction model consists of a Promela model code with an LTL symbol definition file. The major sub-tasks in preprocessing of auction data are illustrated in Figure 4, which consists of 5 steps, namely "Calculate Data Size", "Parse User List", "Re-arrange Data", "Generate Code", and "Generate Symbol Definitions".

\subsection{The Auction Model Template}

The auction model template is written in Promela code. The template allows us to generate different Promela code for auction models based on different extracted numeric auction data.
Table 3: Auction model template code.

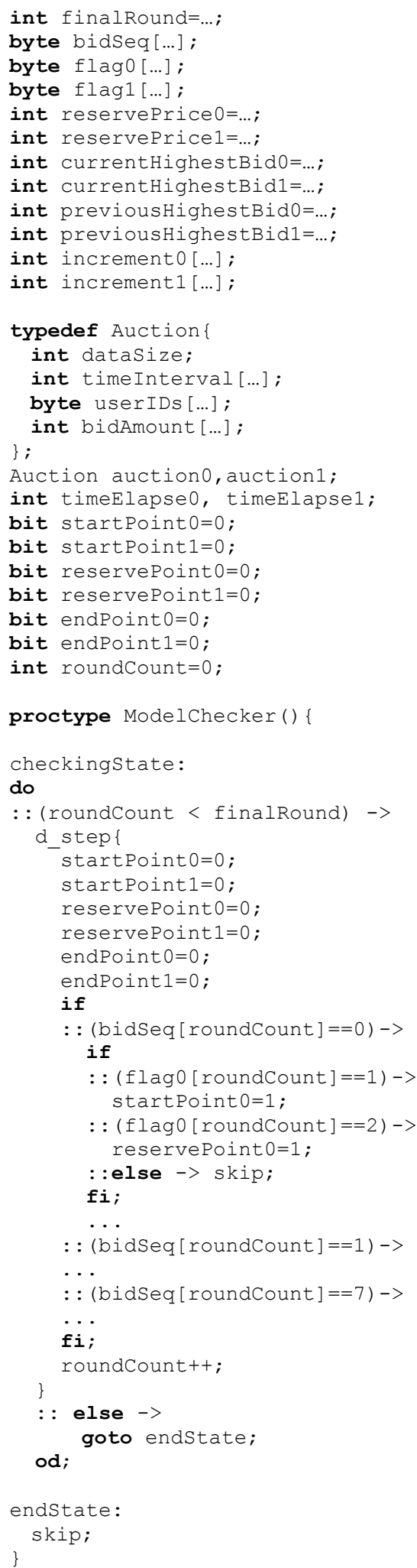




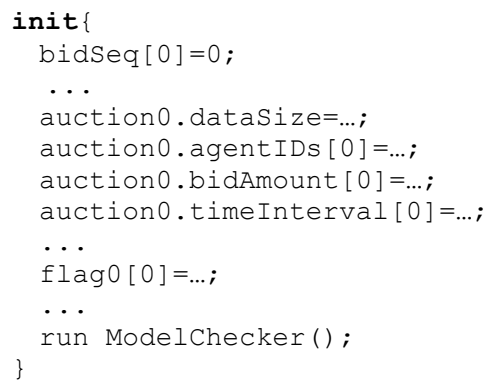

As shown in Table 3, we first define the global variables in the auction template (line 1 19), which will be initialized with values extracted from the auction data in the init procedure (line 67 78). These global variables can be used to define symbols to compose LTL formula. In line 20 28, we define the local variables that can only be used by the model checker.

The code between line 30-66 represents the state transitions of the bidding process. When each auction round starts, all flags are cleared (line 36 41). Then according to different bid sequences that represent different bidding situations, the model runs differently. For example, when the bid sequence is " 0 ", it means that a bidder placed a bid in Auction 0; while at the same time no one was bidding in Auction 1. To handle this case, we first set up the flags, and then all the old values from the previous bid of the relevant variables are updated to the new values that represent the current bid.

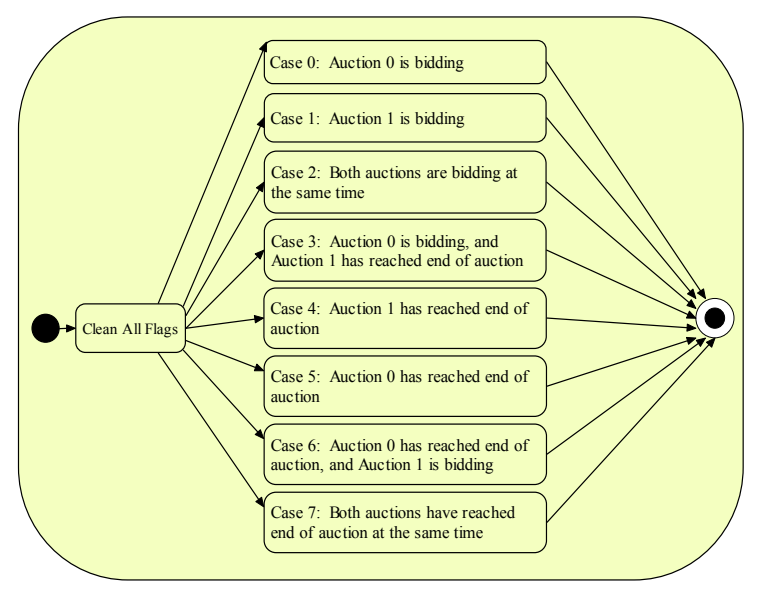

Figure 5: Different bidding situations.

In Figure 5, we show 8 different biding situations in a UML state diagram that correspond to different bidding sequences. Since each auction can be in one of the following states: "bidding", "not biding" and "end", we shall have 9 combinations for two concurrent auctions. However, one of the cases (i.e., "not bidding, not bidding") can be excluded from consideration because when it happens, both auctions must have already ended.

\subsection{Symbol Definitions for LTL}

In order to verify properties specified in LTL formulas, we need to define symbols that can be used in formula composition. We define most of the terms to be self-explanatory. For example, "start0" ("start1") denotes the start of Auction 0 (Auction 1); while "end0" ("end1") denotes the end of Auction 0 (Auction 1). Similarly, the symbol "reserve0" ("reserve1") denotes that the reserve price of Auction 0 (Auction 1) is reached. However, for a term like "bid00", the first " 0 " denotes Auction 0, and the second " 0 " denotes the bidding behavior of User 0. Thus, if a user numbered 16 bids on Auction 0 , then it should be represented by the symbol "bid016".

Symbol definitions, combining with LTL formulas, can ease the task of writing LTL formulas. In practical use, users can also develop their own set of symbol definitions.

\subsection{The Model Checking Process}

After the auction model has been created, the model checking process becomes simple. The model checking process consists of the following steps.

1. Duplicate the symbol definition file called "definitions.txt" and name it as "pan.ltl".

2. Type in the LTL formula. The formula will be translated to "never claim" that can be used to match either finite or infinite behaviors.

3. Append the generated "never claim" to the "pan.ltl" file.

4. Use the SPIN to generate a model verifier (pan) from both the Promela model code ("pan_in") and the file "pan.ltl". The file "pan.ltl" should contain both a "never claim" and symbol definitions.

5. Compile the verifier source code (pan) using "gcc" to produce an executable file "pan.exe".

6. Execute the model verifier. After the execution of the auction model, the model verifier will show whether the result is valid or invalid. An invalid result indicates that during the verification process, the model verifier encountered errors. If any errors are encountered, the model we developed violates the LTL formula that we specified. In this case, the behavior we specified does not exist in the model being checked. 


\section{A CASE STUDY}

In this section, we use a case study to show how potential shills can be detected. The auction data was collected from the eBay, but to protect users' privacy, we have changed all user IDs (due to page limitation, the auction data is not presented in this paper). The titles for the two auctions are the same, which is "HP/COMPAQ PRESARIO LAPTOP CDRW BURNER DVD WIRELESS". Both auctions are held by the same seller and the description to these two auction items is shown in Table 4.

Table 4: Description of auctioned items.

\begin{tabular}{|ll|ll|}
\hline \multicolumn{4}{|l|}{ Item Specifics - PC Laptops } \\
\hline Brand: & \multicolumn{1}{l|}{ Compaq } & Hard Drive Cap: & 60 GB \\
\hline Chip Type: & -- & Screen Size: & 15 inches \\
\hline Model: & -- & OS Included: & Yes \\
\hline $\begin{array}{l}\text { Processor } \\
\text { Speed: }\end{array}$ & $\mathbf{1 . 4} \mathbf{~ G H z}$ & Primary Drive: & $\begin{array}{l}\text { CD-RW/DVD } \\
\text { Combo }\end{array}$ \\
\hline Memory & $\mathbf{5 1 2} \mathbf{~ M B}$ & Condition: & -- \\
\hline
\end{tabular}

To simplify our verification process, we have made a few adjustments for the auction data. We erased all currency symbols and time zone abbreviations to make them appear simpler. We rounded up all bidding prices that have decimals. In addition, we added a user's bidding price by 1 if the user's bidding price is the same as the previous bid. Note that each user name is associated with a numeric value in parentheses, which represents the user's feedback score.

Since the eBay does not provide any information about the reserve price for each auction, as well as whether the seller has set up a reserve price, we assume the reserve prices for both auctions are $\$ 500$. The value of $\$ 500$ is very close to both winning bids (\$630 and \$620), but sill gives us good ranges (from $\$ 500$ to $\$ 630$ and from $\$ 500$ to $\$ 620$ ) for checking bidding behaviors. We define overbid and deliberate bid as follows.

Definition If the price difference between the previous bid and the current bid is over 10 dollars, it is considered as an overbid (a bid in large increment).

Definition If the time gap between the current bid and the previous bid is over 7200 seconds ( 2 hours), it is considered as a deliberate bid.

The reason we set 10 dollars as the boundary is based on our observation, since most bid increments are less than this number. Similarly, we set the 2 hours boundary for deliberate bid because 2 hours is a reasonable period of time for a bidder to make a deliberate decision. In practical, these two values should be defined and adjusted according to auction administrator's experiences and observations.

In the two concurrent online auctions, there are totally 35 users, among which we selected the following four users for investigation: "paperchen", "benniten23", "andy293" and "yass3d". We chose these four users because they are the only bidders who are involved in both of the two concurrent online auctions, and thus, they are more likely to be shills.

After the auction data has been preprocessed, the data is re-arranged. We then determine the auction data's overlapping style as shown in Figure 7.

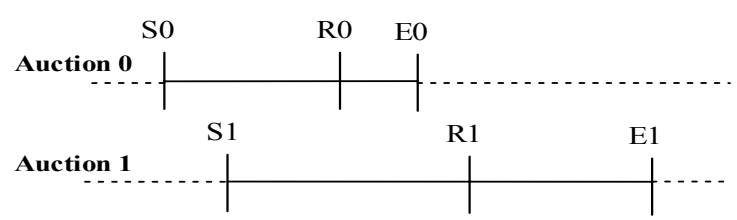

Figure 7: Overlapping style for the two auctions.

We now use model checking techniques to verify the following behavioral properties that are related to shilling behaviors.

Property 1: User places a deliberate overbid before R0 in Auction 0 or before R1 in Auction 1, but doesn't bid at all after R0 or R1.

Patterns Used: (1) Existence, before $R$ (2) Absence, after $Q$

Formulas:

For Auction 0: ( (! reserve0 W ( (action0) \&\& !reserve0) ) ) \&\& ( [ ] (reserve0->

[] (! action 1$))) \quad$ i.e., $((($ !reserve0 U $(($ action $) \& \& !$ reserve0 $))||([]$ !reserve 0$))$ )$\& \&([]($ reserve $0->[]($ !action 1$)))$

For Auction 1: ( (!reserve1 W ( (action 0$) \& \&$ !reserve1) ) ) \&\& ( [ ] (reserve1->

[] (!action 1$)))$ i.e., (( (!reserve1 U $(($ action $) \& \&$ !reserve 1$))||([]$ !reserve 1$))$ )$\& \&([]($ reserve1-> [ ] (!action 1$)))$

Actions: For Auction 0: (overBid06 \&\& deliBid0), (overBid014 \&\& deliBid0), (overBid016 \&\& deliBid0) and (overBid017 \&\& deliBid0) for action0; bid06, bid014, bid016 and bid017 for action1. For Auction 1, the same rule applies.

Note: deliBid0 means the time interval between currently placed bid and previous bid is longer than the limit we have set, so the bid is considered as a deliberate bid. The formula (overBid06 \&\& deliBid0) means "paperchen" (numbered 6) is placing a deliberate overbid. 
Table 5: Model checking results for Property 1.

\begin{tabular}{|c|c|c|}
\hline User & Auction 0 & Auction 1 \\
\hline paperchen (5) & Valid & Valid \\
\hline benniten $23(1)$ & Invalid & Invalid \\
\hline andy293 (12) & Invalid & Invalid \\
\hline yass3d (12) & Invalid & Invalid \\
\hline
\end{tabular}

Explanation: This property implies that a user places an overbid to stimulate the bidding when he notices that it has been a while since previous bid was placed, and he stops bidding after the bid reaches the reserve price. This behavior is highly suspicious for shilling. From Table 5, we find that only "paperchen" has such behavior, so he is very likely a shill.

Property 2: After S1 until E0, user bids in auctions that have higher bidding price.

Patterns Used: Existence, After $Q$ until $R$

\section{Formulas:}

For Auction 0: ( [ ] (start $1 \& \& !$ end0->(! end0 U $(($ action $) \& \&$ !end0) )) )

For Auction 1: ( [ ] (start $1 \& \&$ ! end0-> (!end0 U $(($ action $) \& \& !$ end 0$)))$ )

Actions: (bid06 \&\& p1Lower), (bid014 \&\& p1Lower), (bid016 \&\& p1Lower), (bid017 \&\& p1Lower) for Auction 0. The same rule applies to Auction 1 .

Note: The variable p1Lower means the bidding price in Auction 1 is lower than that in Auction 0. So (bid06\&\&p1Lower) means "paperchen" is bidding on Auction 0 when Auction 1 has lower bidding price.

Table 6: Model checking results for Property 2.

\begin{tabular}{|c|c|c|}
\hline User & Auction 0 & Auction 1 \\
\hline paperchen (5) & Invalid & Valid \\
\hline benniten 23 (1) & Valid & Valid \\
\hline andy293 (12) & Valid & Invalid \\
\hline yass3d (12) & Valid & Invalid \\
\hline
\end{tabular}

Explanation: Since during the time the two auctions overlap, anyone who doesn't bid on the auction that has the cheaper bidding price is suspicious. From Table 6, we notice that the results for the user "benniten23" were valid for both auctions, which suggest that the user might not be a normal bidder.

Based on the above analysis, we can conclude that the user "paperchen" and "benniten23" are possible shills because both of them attempted to drive up the bidding price and one of them ("paperchen") stopped bidding after the price reached the reserve price. When a certain amount of time has passed after the last bid, "paperchen" tried to create a competitive bidding atmosphere by placing overbids. Notice that our approach can effectively detect and suggest shill suspects; however, it is not guaranteed that suspects must be shills. Based on the model checking results, we can easily track the suspects' bidding history and draw conclusions if the suspects are actually shills.

\section{CONCLUSIONS AND FUTURE WORK}

In this paper, we introduced a model checking approach to detecting shilling behaviors in concurrent online auctions. We proposed an auction model template that supports automatic generation of auction models based on auction data. With our pattern-based model checking approach, we can not only easily write specifications for bidding behaviors, but also directly detect suspicious shills in concurrent online auctions. Our approach can be easily extended to support more than two concurrent online auctions. To provide tool support for automatic generation of specifications of shilling behaviors envisions our future research work.

\section{REFERENCES}

Dwyer, M. B., Avrunin, G. S., and Corbett, J. C., 1999. Patterns in property specifications for finite-state verification. In Proceedings of the 21st International Conference on Software Engineering (ICSE 99), Los Angeles, pp. 16-22.

Gupta, A. and Bapna, R., 2002. Online mercantile processes: a closer look at $\mathrm{B} 2 \mathrm{C}$ online auctions. In Handbook of Electronic Commerce in Business and Society, P. B. Lowry, R. J. Watson, and J. O. Cherrington eds., St. Lucie Press, pp. 85-98.

Holzmann, G. J., 1997. The model checker SPIN. IEEE Transactions on Software Engineering, Volume 23, No. 5, pp. 279-295.

Kauffman, R. J., and Wood, C. A., 2000. Running up the bid: modeling seller opportunism in Internet auctions. In Proceedings of the Sixth Americas Conference on Information Systems (AMCIS 2000), M. Chung (ed.), Long Beach, CA, pp. 929-935.

Pnueli, A., 1977. The temporal logic of programs. In Proceedings of the 18th IEEE Symposium on Foundations of Computer Science, IEEE Computer Society Press, pp. 46-67.

Wang, W., Zoltán, H., and Whinston, A. B., 2002. Shill bidding in multi-round online auctions. In Proceedings of the 35th Hawaii International Conference on System Sciences (HICSS'02), Hawaii, USA. 